\title{
Evaluar el Pensamiento Computacional mediante Resolución de Problemas: Validación de un Instrumento de Evaluación
}

\section{Evaluating Computational Thinking through Problem Solving: Validation of an Assessment Instrument}

\author{
Beatriz Ortega-Ruipérez ${ }^{1,{ }^{*}}$ y Mikel Asensio ${ }^{2}$ \\ ${ }^{1}$ Universidad Internacional de La Rioja, España \\ ${ }^{2}$ Universidad Autónoma de Madrid, España
}

\begin{abstract}
DESCRIPTORES:
Resolución de problemas

Instrumento de

evaluación

Pensamiento computacional

Validez

Educación permanente

\section{RESUMEN}

El pensamiento computacional se está evaluando, en la mayoría de los enfoques, a través de elementos de programación. Desde aquí se promueve un enfoque de evaluación desde la resolución de problemas complejos, ya este pensamiento es utilizado como una estrategia de resolución de problemas. En este artículo se valida el constructo teórico de un instrumento de evaluación para medir pensamiento computacional mediante la resolución de problemas complejos, con una batería de pruebas compuesta por 15 ítems. En primer lugar, se describen los principios utilizados para el diseño, principios basados en el enfoque de evaluación de sistemas complejos múltiples y el marco de PISA utilizado en 2012. Posteriormente, se analiza el modelo teórico propuesto de 2 factores: representación del problema y resolución del problema, y varios modelos adicionales con ajustes a partir del modelo teórico. Se determina que el modelo que mejor ajusta es el de 2 factores, coincidiendo con la propuesta teórica. Por último, se realizan análisis, por un lado, de la adecuación de los ítems a cada factor, confirmando asíla adecuación de las pruebas, y por otro lado, la correlación entre factores obteniendo un 0,969 . Se concluye que el instrumento tiene un grado de validez muy elevado, por lo que resulta adecuado para medir el pensamiento computacional a través de la resolución de problemas.
\end{abstract}

\section{KEYWORDS: \\ Problem solving \\ Assessment instrument \\ Computational thinking \\ Validity \\ Lifelong learning}

\begin{abstract}
:
Computational thinking is being assessed, in most approaches, through elements of programming. From here, an evaluation approach is promoted from the resolution of complex problems, as this thinking is used as a problem-solving strategy. This article validates the theoretical construct of an assessment instrument to measure computational thinking through complex problem solving, with a test battery composed of 15 items. First, the principles used for the design are described, principles based on the multiple complex systems assessment approach and the PISA framework used in 2012. Subsequently, the proposed 2 -factor theoretical model is discussed: problem representation and problem resolution, and several additional models with adjustments from the theoretical model. It is determined that the best fitting model is the 2 -factor model, coinciding with the theoretical proposal. Finally, analyses are made, on the one hand of the suitability of the items to each factor, thus confirming the suitability of the items, and on the other hand, the correlation between factors, obtaining a 0.969. It is concluded that the instrument has a very high degree of validity, so that it is suitable for measuring computational thinking through problem solving.
\end{abstract}

CÓMO CITAR:

Ortega-Ruipérez, B. y Asensio, M. (2021). Evaluar el pensamiento computacional mediante resolución de problemas: validación de un instrumento de evaluación. Revista Iberoamericana de Evaluación Educativa, 14(1), 153-171. https://doi.org/10.15366/riee2021.14.1.009

${ }^{*}$ Contacto: beatriz.ortega.ruiperez@unir.net

ISSN: 1989-0397

https://revistas.uam.es/riee
Recibido:

28 de abril 2020

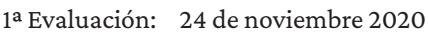

2a Evaluación: 4 de diciembre 2020

Aceptado: 


\section{Introducción}

La evaluación de competencias para la vida debe ser un asunto prioritario en la educación para conocer cómo debemos planificar el proceso de enseñanza, de cara a conseguir que nuestros alumnos puedan lograr una educación integral. En este sentido, Román y Murillo (2009) plantean al menos tres exigencias a la evaluación: que permita validar y reforzar el desarrollo integral, ya que en la educación se prioriza lo que se evalúa; que se contextualicen los aprendizajes medidos gracias a la comprensión de factores que componen un aprendizaje significativo, algo que se puede ver facilitado por el alto desarrollo tecnológico; y que se incluyan criterios y estrategias para mejorar la aplicación de la evaluación, ya que con unos resultados fiables sobre un aprendizaje se puede mejorar el proceso de enseñanza sobre éste.

Por ello, para evaluar el pensamiento computacional no podemos reducirnos a evaluar prácticas de programación, pues no estaremos seguros de si lo que evaluamos es el pensamiento o la capacidad para crear programas. Por ello, se promueve un enfoque de evaluación desde la resolución de problemas complejos, ya que este pensamiento es utilizado como una estrategia de resolución de problemas.

\subsection{Pensamiento computacional}

El pensamiento computacional (PC) (Wing, 2006) es un concepto que surge desde las Ciencias de la Computación, propuesto como unas habilidades que permite abordar los problemas complejos de forma computacional. Por tanto, a partir de esta definición podemos afirmar que se trata de un concepto que define una estrategia de pensamiento.

En su origen se definió como un pensamiento que influye en la planificación y ejecución de los problemas complejos; al utilizar técnicas que emplean habitualmente los científicos de la computación, facilitando la resolución de estos. En este caso, el PC se emplea como un pensamiento estratégico basado en un enfoque computacional de cara al establecimiento de un plan (Schoppek, 2002).

Posteriormente, surgen evidencias a favor (Kwisthout, 2012; Wing, 2014) de que es necesario un modelo computacional para la representación de problema complejos, ya que sería más fácil gestionar gran cantidad de variables consideradas de una relevancia similar entre ellas, y se facilitaría así la resolución de problemas. En este caso, el pensamiento computacional se emplea como un modelo de procesamiento de información, una estrategia de pensamiento de nivel superior que es capaz de computar un gran modelo para facilitar su representación.

Por tanto, las investigaciones posteriores sugieren que también se utiliza una estrategia computacional para construir esa representación, por lo que podríamos decir que el PC también facilita la representación de problemas complejos, y no sólo se aplica al proceso de resolución como se había planteado inicialmente.

Así, se pretende partir de una definición adecuada de este pensamiento, ya que, al igual que sugieren Blackwell et al. (2008), es importante conocer las ventajas de adoptar este nuevo estilo de pensamiento en la resolución de problemas, de modo que realmente aporte valor. Hasta ahora se suele aplicar en el diseño de programas, en ciencias de la computación (Hsu et al., 2018), pero apenas existen propuesta desde la resolución de problemas (Ortega-Ruipérez y Asensio, 2018). De hecho, en muchas ocasiones se pretende trabajar el PC desde la programación, sin mencionar que se trabaja ni cómo se hace (Heintz et al., 2016).

Este es un error común que se ha extendido de la enseñanza del PC a su evaluación. Como se puede apreciar en diferentes enfoques e instrumentos de evaluación del PC, lo que realmente se está evaluando es el conocimiento en conceptos propios de la programación (Brennan y Resnick, 2012; Koh et al., 2014; Román-González et al., 2018), pero no se evalúan procesos cognitivos propios de un pensamiento en la resolución de problemas.

De hecho, Lye y Koh (2014) realizan una revisión de la evaluación del PC desde la programación, y descubren que estos instrumentos ni siquiera evalúan las prácticas definidas para el PC, propuestas por Brennan y Resnick (2012), sino que se limitan a evaluar los conceptos del PC (para más información, revisar Ortega-Ruipérez, 2018).

En una revisión de la literatura sobre cómo se aborda la evaluación del PC, Tang et al. (2020) comprueban que sólo el 9,8\% de las herramientas de evaluación del PC no tienen relación con STEM (ciencias, tecnología, ingeniería y matemáticas). Además, el 68,5\% de las evaluaciones se realizaban a través de la programación y la robótica. Y un 21,7\% eran STEM, pero no trataban sobre programación y robótica. 
Por tanto, se reclama la necesidad de la creación de un instrumento de evaluación que ofrezca una forma de comprobar si mejora el pensamiento computacional al resolver problemas complejos, aplicados a cualquier ámbito, y no solo respecto a la programación y el diseño de programas informáticos.

\subsection{Resolución de problemas complejos}

En la otra parte de esta investigación, por tanto, se encuentra la resolución de problemas complejos (RPC), cuyo enfoque de investigación se ha ido distanciando de la investigación de resolución de problemas tradicional, debido a las características propias que contienen este tipo de problemas.

Funke y Frensch (1995) proponen una definición de los problemas complejos que suponen a la persona que lo resuelve unos requisitos concretos para poder ser resueltos. Principalmente estas peculiaridades residen en que, para resolver problemas complejos, la persona no dispone de todos los datos del contexto desde el inicio del problema. Este motivo hace que necesite evaluar continuamente cada estado del problema en el que se encuentra desde que parte del estado inicial, para recoger nuevos datos y valorar si avanza hacia el estado objetivo o debe reformular el problema debido a que cambia su representación sobre éste. Al no tener los datos desde el inicio del problema, los problemas complejos también se denominan problemas dinámicos, diferentes a los problemas estáticos (Herde et al., 2016).

Las características particulares que definen a los problemas complejos requieren estrategias más elaboradas que las empleadas tradicionalmente en la resolución de problemas, para enfrentarse a este tipo de problemas de forma que logremos resolverlos. De ello se deduce que es fundamental estudiar qué estrategias son más adecuadas para abordar problemas complejos. Y para ello, debemos generar instrumentos de evaluación diferentes a los utilizados tradicionalmente para el estudio de resolución de problemas, para investigar cómo se estructura la RPC.

La RPC, como tema de investigación independiente, se ha empezado a estudiar con más fuerza durante estos últimos años. Como recoge Funke (2013), la investigación sobre resolución de problemas ha aumentado durante los últimos años, y con la llegada de nuevos métodos para el rastreo de procesos, la creación de micromundos virtuales y la definición de nuevos constructos, las teorías están siendo ajustadas.

Inicialmente, Funke (2001) propuso los enfoques originales MicroDYN y MicroFIN, siendo uno de ellos cuantitativo y el otro cualitativo, respectivamente, para la evaluación de la RPC. El enfoque de las pruebas MicroDYN se basa en el marco de sistemas lineales de ecuaciones estructurales (LSE), mientras que el enfoque de MicroFIN se basa en el marco de sistemas autómatas de estados finitos (FSA).

Más recientemente, Greiff et al. (2012) han propuesto un enfoque de sistemas complejos múltiples, que aúna ambos marcos, y que permite aumentar su complejidad en cuanto a la evaluación (Herde et al., 2016). Su propuesta consiste en sistemas informáticos que permitan reproducir y medir la interacción entre el problema y la persona, de una forma más sencilla y fiable que las pruebas de lápiz y papel.

Las tres ventajas principales del enfoque de sistemas complejos múltiples son: (1) permite discriminar varios niveles de competencia, (2) estima con una alta fiabilidad la adquisición del conocimiento y la aplicación del conocimiento, y (3) limita dificultades en las puntuaciones finales de RPC debidas a errores aleatorios aislados al comienzo de la evaluación (Herde et al., 2016).

Este enfoque resulta adecuado para el estudio del empleo de estrategias de nivel superior en la resolución de problemas complejos, ya que nos permite distinguir entre una primera fase de adquisición del conocimiento sobre el problema y una segunda fase de aplicación del conocimiento para el control del problema (Funke, 2001).

La mejora de la evaluación con el uso de test informatizados ya se viene prediciendo desde los años noventa. Olea y Ponsoda (1998) ya hablaban de que los avances psicométricos y los avances informáticos estaban causando una auténtica revolución en el diseño de pruebas psicológicas y educativas, ya que, la convergencia entre ambos campos incrementa el valor diagnóstico de las pruebas de evaluación, al medir el rendimiento de procesos cognitivos implicados en la resolución de estas.

Este enfoque se incorporó parcialmente en las tareas utilizadas para la evaluación de la RPC en el Programme for International Student Assessment (Herde et al., 2016), o programa PISA, en su edición de 2012, ya que se basó en pruebas digitales en las que los alumnos interaccionan de forma continua con los datos de problemas dinámicos. 
Las pruebas creadas para este enfoque pretenden tener conexión con temas de la vida real, intentando con ello superar las limitaciones que detectaron Gallardo-Gil, et al. (2010) sobre el enfoque de PISA 2006, enfoque que consideran haberse restringido a pruebas con temas académicos. Además, este enfoque reduce las limitaciones metodológicas que han mostrado las pruebas PISA en ediciones anteriores, como ha mostrado análisis de estas pruebas (González-Mayorga et al., 2017).

Por todo lo expuesto, resulta necesario investigar sobre cómo evaluar si el pensamiento computacional facilita la resolución de problemas complejos. Aquí se propone y analiza psicométricamente una herramienta que pretende ayudar a avanzar en la evaluación del pensamiento computacional más allá de las prácticas de programación, contexto en el que desarrollan dicho pensamiento, y que, por tanto, no permite generalizar.

\section{Método}

\subsection{Objetivosy diseño}

El objetivo de esta investigación es validar el constructo teórico de un instrumento de evaluación de la resolución de problemas complejos en español, que sirve para medir estrategias de RPC como la estrategia computacional. Para ello, se concretan dos objetivos específicos.

El primer objetivo específico es comprobar si el modelo teórico planteado de 2 factores es el modelo con el que el instrumento es el que mejor describe el instrumento. Para este objetivo se parte de una propuesta teórica de 4 fases en la resolución de problemas (explorar y comprender, representar y formular, planificar y ejecutar, observar y reflexionar), que asimismo se pueden agrupar en 2 fases o factores que implican diferentes procesos cognitivos (representación del problema las dos primeras y resolución del problema las dos últimas). Para confirmar el objetivo se van a comparar diferentes modelos, con diferente número de factores a considerar $(1,2$ y 4) y haciendo a su vez distintos ajustes en cada modelo.

El segundo objetivo específico es profundizar en la adecuación de los ítems en el modelo finalmente seleccionado, de cara a confirmar que el instrumento es adecuado según la propuesta teórica, y por tanto se puede confirmar que mide lo que pretende.

Para responder a estas cuestiones se utiliza el método de estimación ULSMV, método que produce errores estándar estimados más precisos de las cargas factoriales para ítems ordinales categóricos y tamaños muestrales reducidos (Li, 2014).

\subsection{Población y Muestra}

Los participantes de la investigación son dos muestras de alumnos de $4^{\circ} \mathrm{ESO}$, en total 66 participantes. Los participantes tienen 15 años, edad para la que ha sido diseñado el marco de PISA 2012, y que se ha tomado de referencia para la creación del instrumento de evaluación. Del total de los participantes, 28 de ellos son alumnos de un centro concertado de Valdemoro, Madrid, y los otros 38 participantes pertenecen a una Escuela de Las Rozas, Madrid.

El instrumento pretende ser válido para evaluar la capacidad de resolver problemas complejos en cualquier sujeto de 15 años que tenga como lengua materna el español, ya que la prueba requiere su total comprensión para poder eliminar el posible efecto.

\subsection{Instrumento}

El diseño de las pruebas del instrumento se enmarca en el programa PISA mide diferentes aspectos en alumnos de 15 años a nivel internacional, permitiendo realizar comparaciones entre los diferentes países participantes, de cara a detectar puntos débiles en los sistemas de enseñanza y así mejorarlos (Schleicher, 2006). En la edición de PISA 2012 se incluyen pruebas de RPC, basadas en el enfoque de sistemas complejos múltiples. Pero no se mantienen en ediciones posteriores. En la edición de PISA 2015, se evalúa la resolución de problemas de forma colaborativa, adoptando un marco diferente (Greiff et al., 2013).

En este caso, vamos a utilizar el marco de evaluación PISA 2012 (OECD,2010), que se puede ver en el Cuadro 1, para crear pruebas que conformen nuestro instrumento de evaluación, ya que este marco adopta las caracte- 
rísticas de evaluación de los sistemas múltiples complejos (MCS), que supone mejoras respecto a otros enfoques de evaluación. Por tanto, según las características de los MCS, se han desarrollado pruebas informáticas con situaciones en las que se tiene que interactuar con el problema.

Por tanto, el marco utilizado permite la evaluación del uso de la estrategia computacional en la RPC, ya que la estrategia computacional, también denominada pensamiento computacional, se aplica en la resolución de problemas complejos al descomponerlos en problemas más sencillos de abordar, por lo que estas pruebas nos permitirán conocer la relación entre ambos.

Cuadro 1

Instrumento PISA 2012 para la resolución de problemas complejos (OECD, 2010), basado en el enfoque de sistemas múltiples complejos (Greiffet al., 2012)

\begin{tabular}{|c|c|c|c|c|}
\hline & Explorar y comprender & Representar y formular & Planear y ejecutar & Observar y reflexionar \\
\hline 1 & $\begin{array}{l}\text { Pueden explorar de for- } \\
\text { ma limitada, si detectan } \\
\text { algo muy similar. }\end{array}$ & $\begin{array}{l}\text { Sabe cómo funciona un } \\
\text { dispositivo simple, si de- } \\
\text { tecta algo muy similar. }\end{array}$ & $\begin{array}{l}\text { Resuelven problemas si } \\
\text { hay una condición simple } \\
\text { y pocos pasos para el } \\
\text { objetivo. }\end{array}$ & $\begin{array}{l}\text { Tienden a no planificar } \\
\text { con anticipación o esta- } \\
\text { blecer subobjetivos. }\end{array}$ \\
\hline 2 & $\begin{array}{l}\text { Pueden explorar un es- } \\
\text { cenario de un problema } \\
\text { no familiar y entender } \\
\text { una pequeña parte. }\end{array}$ & $\begin{array}{l}\text { Algunas veces, logran } \\
\text { entender y controlar } \\
\text { dispositivos digitales con } \\
\text { controles no familiares. }\end{array}$ & $\begin{array}{l}\text { Pueden probar una } \\
\text { hipótesis simple y resolv- } \\
\text { er un problema con una } \\
\text { restricción específica. }\end{array}$ & $\begin{array}{l}\text { Pueden planificar y ejecu- } \\
\text { tar un solo paso a la vez } \\
\text { para lograr un subobjeti- } \\
\text { vo, y tienen cierta capaci- } \\
\text { dad para monitorizar el } \\
\text { proceso. }\end{array}$ \\
\hline 3 & $\begin{array}{l}\text { Pueden inferir rela- } \\
\text { ciones simples entre } \\
\text { variables. Con condi- } \\
\text { ciones interrelaciona- } \\
\text { das, dejan una variable } \\
\text { constante para ver el } \\
\text { cambio en otras. }\end{array}$ & $\begin{array}{l}\text { Pueden manejar infor- } \\
\text { mación presentada en } \\
\text { varios formatos. Pueden } \\
\text { controlar dispositivos } \\
\text { digitales simples, pero } \\
\text { tiene problemas para más } \\
\text { complejos. }\end{array}$ & $\begin{array}{l}\text { Pueden tratar con una } \\
\text { condición, por ejemp- } \\
\text { lo, para generar varias } \\
\text { soluciones y chequear } \\
\text { cuál de ellas satisface la } \\
\text { condición. }\end{array}$ & $\begin{array}{l}\text { Pueden idear y ejecutar } \\
\text { pruebas para confirmar } \\
\text { o refutar hipótesis. Ellos } \\
\text { entienden la necesidad } \\
\text { de planear y monitorizar } \\
\text { el progreso, y son capaces } \\
\text { de intentar una opción } \\
\text { diferente si es necesario. }\end{array}$ \\
\hline 4 & $\begin{array}{l}\text { Pueden explorar un } \\
\text { problema bastante } \\
\text { complejo. Entienden la } \\
\text { relación entre sus com- } \\
\text { ponentes. }\end{array}$ & $\begin{array}{l}\text { Pueden hipotetizar por } \\
\text { qué un sistema funciona } \\
\text { mal, y cómo probarlo. } \\
\text { Pueden dominan disposi- } \\
\text { tivos bastante complejos. }\end{array}$ & $\begin{array}{l}\text { Pueden probar sistemáti- } \\
\text { camente posibilidades } \\
\text { y chequear si múltiples } \\
\text { condiciones se satisfacen. }\end{array}$ & $\begin{array}{l}\text { Pueden planear unos po- } \\
\text { cos pasos y monitorizar } \\
\text { el progreso. Suelen ser } \\
\text { capaces de ajustar sus } \\
\text { planes en función del } \\
\text { feedback. }\end{array}$ \\
\hline 5 & $\begin{array}{l}\text { Pueden explorar } \\
\text { sistemáticamente un } \\
\text { problema complejo } \\
\text { para entender cómo se } \\
\text { estructura lo relevante. }\end{array}$ & $\begin{array}{l}\text { Cuando se enfrente a un } \\
\text { dispositivo complejo y } \\
\text { no familiar, responden } \\
\text { rápidamente al feedback } \\
\text { para control el dispositivo. }\end{array}$ & $\begin{array}{l}\text { Para conseguir una solu- } \\
\text { ción, piensan de forma } \\
\text { anticipada para encontrar } \\
\text { la mejor estrategia que } \\
\text { aborda todas las restric- } \\
\text { ciones dadas. }\end{array}$ & $\begin{array}{l}\text { Pueden ajustar inmediat- } \\
\text { amente sus planes cuan- } \\
\text { do detectan dificultades } \\
\text { inesperadas o cuando } \\
\text { cometen errores que les } \\
\text { sacan del camino. }\end{array}$ \\
\hline 6 & $\begin{array}{l}\text { Pueden explorar es- } \\
\text { tratégicamente para } \\
\text { entender un problema, } \\
\text { y la información puede } \\
\text { estar en varios formatos } \\
\text { que requieran inter- } \\
\text { pretación. }\end{array}$ & $\begin{array}{l}\text { Desarrollan modelos } \\
\text { mentales completos y de } \\
\text { varios escenarios, para re- } \\
\text { solverlos de forma eficaz. } \\
\text { Si la tecnología compleja } \\
\text { no les es familiar, apren- } \\
\text { den de forma óptima a } \\
\text { controlarla. }\end{array}$ & $\begin{array}{l}\text { Pueden establecer } \\
\text { hipótesis generales sobre } \\
\text { un sistema y probarlo } \\
\text { a fondo. Pueden guiar } \\
\text { una premisa a través de } \\
\text { una conclusión lógica o } \\
\text { reconocer cuando falta } \\
\text { información. }\end{array}$ & $\begin{array}{l}\text { Para lograr una solución, } \\
\text { pueden crear planes } \\
\text { complejos, flexibles y } \\
\text { de múltiples pasos que } \\
\text { monitorizan de forma } \\
\text { continua. Cuando es } \\
\text { necesario, modifican sus } \\
\text { estrategias, según todo } \\
\text { tipo de restricciones. }\end{array}$ \\
\hline
\end{tabular}

Nota. Elaboración propia.

El instrumento de evaluación se compone de varias pruebas diferentes que permiten evaluar esta competencia en diferentes contextos, de forma que se pueda reducir la posibilidad de que sea el efecto del contexto el que afecte al resultado en lugar del nivel de competencia real. Se emplean tanto problemas personales como socia- 
les, y tecnológicos como no tecnológicos, como veremos más adelante. En el marco PISA 2012 (OECD, 2010), las pruebas discriminan varios niveles de competencia, y en este caso concreto, son seis niveles de competencia.

Además, recoge la evaluación de diferentes fases de la competencia de resolución de problemas. De esta forma, cada una de las fases presenta unos requisitos concretos para cada uno de los niveles de competencia (Cuadro 1), siendo la base para la creación de estas pruebas. De las cuatro fases, las dos primeras fases se corresponden con la adquisición del conocimiento, mientras que las dos segundas fases se corresponden con la aplicación del conocimiento, que pueden estudiarse con alta fiabilidad desde el enfoque de sistemas complejos múltiples (Herde et al., 2016).

- Adquisición: La primera de las fases de la resolución de problemas corresponde con la exploración y comprensión del problema, explorar la situación para entender la relación entre los diferentes elementos o variables del problema. La segunda fase consiste en la representación y formulación del problema, de cara al establecimiento de hipótesis sobre cómo es el problema y cómo van a poder resolverlo.

- Aplicación: La tercera fase de resolución de problemas es la planificación y ejecución del plan para resolver el problema, que parte de la representación que nos hayamos hecho del mismo. La cuarta y última fase es la observación y reflexión sobre si los pasos que estamos llevando a cabo nos están conduciendo a la resolución del problema, o si por el contrario las acciones que realizamos nos alejan de su resolución.

Para cubrir la validez de criterio en el instrumento, se han utilizado dos pruebas empleadas en la propia evaluación de PISA 2012, que han sido liberadas, de forma que no todas las pruebas son de elaboración propia. Las pruebas de PISA escogidas son Climatizador y Billetes. La primera pertenece al marco de LSE, al que pertenece MicroDYN; y la segunda al marco de FSA, al que pertenece MicroFIN.

Respecto a las pruebas creadas que terminan de conformar nuestro instrumento de evaluación, tres de estas pruebas tienen un enfoque cualitativo, relativo por tanto al enfoque de MicroFIN, y son Vídeo, Agenda e Tren. La cuarta prueba tiene un enfoque cuantitativo, basado en MicroDYN, y es la prueba Coche.

En total hay seis preguntas del enfoque MicroFIN frente a cuatro preguntas del enfoque MicroDYN, lo que reduce el posible desequilibrio que podría provocar si existiesen diferencias en los resultados de ambos enfoques. Si, además, sumamos las preguntas de las pruebas extraídas de PISA, serían un total de nueve preguntas del enfoque MicroFIN frente a seis preguntas del enfoque MicroDYN.

Como se puede ver en el Cuadro 2, para crear una batería de pruebas equilibrada, cada una de las preguntas de estas pruebas evalúan una fase diferente en un nivel distinto o en varios niveles distintos según los resultados. Consideramos que no es necesario incluir pruebas del nivel 1, por lo que se evalúan los niveles del 2 al 6.

Cuadro 2

Relación entre las pruebas y las diferentes fases de RPC en los niveles evaluados

\begin{tabular}{lcllll}
\hline & \%* & \multicolumn{1}{c}{ Fase 1 } & \multicolumn{1}{c}{ Fase 2 } & \multicolumn{1}{c}{ Fase 3 } & Fase 4 \\
\hline 2 & $60-100$ & Billetes 2. Parcial & Agenda 1. Parcial & Agenda 2 & Vídeo 2. Parcial \\
3 & $40-70$ & Vídeo 1 & Climatizador 1. & Billetes 1 & Vídeo 2. \\
Completa
\end{tabular}

Notas: * Porcentaje esperado de aciertos

Elaboración propia.

Además, en el Cuadro 2 se puede ver cuál es el porcentaje esperado de aciertos para cada nivel de competencia, en base a los resultados obtenidos en PISA 2012 y al marco teórico de requisitos para cada nivel en 
cada una de las fases (Cuadro 1). Por último, durante el diseño de las pruebas, también se ha mantenido un equilibrio en el contexto que ofrece cada prueba (Cuadro 3).

Cuadro 3

Contexto de las pruebas en función de si es o no es tecnológico, o si es personal o social

\begin{tabular}{|c|c|c|c|c|}
\hline \multirow{2}{*}{ Prueba } & \multicolumn{4}{|c|}{ Contexto } \\
\hline & Tecnológico & No tecnológico & Personal & Social \\
\hline Climatizador & $\mathrm{x}$ & & $\mathrm{X}$ & \\
\hline Billetes & $\mathrm{X}$ & & & $\mathrm{X}$ \\
\hline Vídeo & & $\mathrm{x}$ & & $\mathrm{X}$ \\
\hline Agenda & & $\mathrm{X}$ & $\mathrm{x}$ & \\
\hline Tren & & $\mathrm{X}$ & & $\mathrm{x}$ \\
\hline Coche & $\mathrm{X}$ & & $\mathrm{X}$ & \\
\hline
\end{tabular}

Nota. Elaboración propia.

Las pruebas han sido creadas en base a este marco. Los niveles y las diferentes fases permiten obtener una definición precisa, ya que este marco se basa en investigaciones con larga trayectoria sobre el tema (OECD, 2010).

Los principales autores de la RPC, o dinámicos (Funke, 2001; ver más en Herde et al., 2016), han profundizado en el campo para demostrar la suficiencia de evaluación de este instrumento, que se basa en la existencia y la importancia de procesos generales de resolución de problemas, independientes de dominio (Greiff et al., 2013).

La existencia de estos procesos generales de resolución de problemas se basa en el empleo de estrategias como la que queremos estudiar, la estrategia computacional, que es aplicable a cualquier dominio. El empleo de este tipo de estrategias independientes de dominio es especialmente útil para la resolución de problemas complejos. De esta forma, el enfoque en el que se basa el diseño del instrumento permite comprobar la efectividad del uso de la estrategia computacional en cuanto a la RPC.

Por tanto, el empleo de estos procesos generales es útil en todos los contextos, y especialmente útiles para estructurar problemas en situaciones desconocidas, hacen que el diseño de esta investigación se base en un constructo ampliamente validado.

Las pruebas se aplican en formato digital, ya que requieren de interacción dado el marco de sistemas complejos múltiples y están disponible como archivo a ejecutar (.exe) tanto en Windows como en Linux. Las pruebas están accesibles en el enlace https://bit.ly/2JaucjC, y cada ítem está explicado al detalle en Ortega-Ruipérez (2018). Las imágenes de las pruebas se encuentran anexados como Apéndice.

\subsection{Procedimiento de recogida y análisis de datos}

Algunas de las pruebas elaboradas se emplearon en una prueba piloto que se realizó en formato papel durante el curso 2015/2016, de cara a comprobar dudas en la interpretación de las pruebas y mejorar su redacción. Estas pruebas están basadas en el marco de sistemas autómatas de estados finitos (FSA), es decir, las pruebas con las que se interactúa de forma cualitativa, bajo el enfoque MicroFIN: agenda, vídeo y tren.

Con algunas adaptaciones en función del feedback y en base al marco en el que se fundamenta PISA 2012 para hacer de ellas pruebas interactivas, es decir, que se tenga que interactuar con el problema, se ha creado esta versión computarizada de las pruebas para hacer esta validación del constructo teórico antes de utilizarlas en la investigación principal.

La recogida de datos se ha hecho en dos fases. En una primera fase se recogieron y analizaron los datos de la muestra que se ha utilizado exclusivamente para la investigación (28 participantes). Se realizaron 
análisis descriptivos del porcentaje de aciertos por pregunta para comprobar que se sitúan en el nivel de competencia esperado.

Una vez se dio por suficiente una primera estadística descriptiva de las pruebas, se recogieron los datos de la segunda muestra $(n=38)$ para contrastar estos análisis descriptivos entre ambas muestras, de cara a comprobar que no hay diferencias significativas entre ambas muestras y que la muestra total arroja resultados similares. Inicialmente esta segunda muestra iba a ser mayor, contando con otros 2 centros con un número similar de sujetos. Sin embargo, la supervisión de la enseñanza de programación no se pudo dar lo suficiente para asegurar que los alumnos aprendían programación de una forma productiva y no reproductiva (Zapata-Ros, 2015). Finalmente, se cuenta en total con un número de participantes ajustado pero suficiente $(n=66)$ que permite realizar análisis de validación del constructo teórico a través del método de estimación ULSMV para ítems ordinales y tamaños muestrales reducidos.

\subsection{Análisis de datos}

Para conocer qué modelo factorial es el más adecuado para explicar este instrumento, se analizan diferentes estadísticos que nos proporciona el método de estimación ULSMV. Para ello se ha utilizado el programa estadístico Mplus.

El primer estadístico analizado es el Chi-Cuadrado, que somete al modelo a un contraste en el que supone que el modelo es igual a un modelo donde no existe correlación en ninguna variable, donde un alto valor de Chi-Cuadrado predice que el modelo no se adecúa bien. Por tanto, cuanto menor sea el estadístico, mejor predice el modelo al instrumento. Asimismo, el P-Valor del estadístico debe resultar no significativo para representar un buen ajuste del modelo.

El segundo estadístico analizado es el índice de error de aproximación cuadrático medio (RMSEA), un índice fiable para evaluar el ajuste general del modelo cuando éste no tiene error de especificación. Este estadístico tiene un intervalo de confianza del $90 \%$. Se considera aceptable cuando es inferior a 0,08 y bueno cuando es inferior a 0,05.

El tercer estadístico es el índice de ajuste comparativo (CFI) que nos permite conocer si el modelo se ajusta adecuadamente a los datos, considerando un buen modelo a partir de 0,95. Este estadístico se debe tener en cuenta junto a otros. Por ello, el cuarto y último estadístico es el índice de Tucker-Lewis (TLI), con un objetivo similar al estadístico anterior, para adoptar una estrategia de evaluación por varios índices, que permita tomar una decisión más fiable, ya que los dos índices deben lograr buenos resultados para seleccionar un modelo concreto, debiendo estar el TLI también por encima de 0,95.

Para seleccionar los modelos a comparar se ha elegido el índice RMSEA, eligiendo únicamente los modelos con un 0,05 como máximo, obteniendo en total 6 modelos.

Los modelos 1 y 2 son modelos de 2 factores, correspondiendo el primero a la representación del problema (incluyendo las fases explorar y comprender; y representar y formular) y el segundo a la resolución del problema (incluyendo las fases planificar y ejecutar; y observar y reflexionar). En el modelo 1 se han fijado todos los errores entre ítems de las mismas preguntas a 0, para eliminar la correlación entre los errores de estimación, ya que habría una correlación muy alta si no que podría afectar al modelo. En el modelo 2 , además de tener todos los errores entre ítems de las mismas preguntas fijados en 0 , se ha fijado a 0 los errores entre los ítems coche $4 \mathrm{y}$ tren 2, por sugerencias del modelo anterior, y que podría estar afectando al modelo.

Los modelos 3 y 4 son modelos de 4 factores, correspondiendo cada factor a cada una de las fases definidas en la teoría sobre RPC: explorar y comprender, representar y formular, planificar y ejecutar, observar y reflexionar. En el modelo 3 se han fijado todos los errores entre ítems de las mismas preguntas a 0 . En el modelo 4 , además de tener todos los errores entre ítems de las mismas preguntas fijados en 0 , se ha fijado a 0 los errores entre los ítems coche $4 \mathrm{y}$ tren 2 por el mismo motivo que antes.

Los modelos 5 y 6 son modelos de 1 factor, considerando todos los ítems de las pruebas pertenecientes a una única dimensión de resolución de problemas complejos. En el modelo 5 se han fijado todos los errores entre ítems de las mismas preguntas a 0 . En el modelo 6 se han eliminado los ítems que en el modelo 5 han obtenido una escasa o nula proporción en la varianza explicada por cada ítem, obteniendo un R-Cuadrado estimado menor al 10\% y con un P-Valor superior a 0,2. 
Una vez confirmado si el modelo teórico inicial es el más adecuado, se profundiza en la adecuación de los ítems explorando los pesos factoriales de los mismos, la correlación entre los factores, la proporción de varianza explicada por ítem con R-Cuadrado y la estimación de dificultad de los ítems mediante la teoría de respuesta al ítem (TRI), concretamente se utiliza el modelo de Rasch para ítems dicotómicos y el modelo de crédito parcial de Masters para el resto de ítems, con 3 puntuaciones (Huggins-Manley y Algina, 2015).

\section{Resultados}

\subsection{Comparación de modelos factoriales}

Los resultados de todos los estadísticos a analizar en cada uno de los 6 modelos se encuentran en el Cuadro 4. Como se puede observar, el modelo 1 es el modelo que presenta las mejores puntuaciones en CFI $(0,979)$ y en TLI $(0,974)$, siendo las únicas puntuaciones en estos estadísticos que superan el valor de 0,95 necesario para considerar que es un buen modelo.

Además, en el modelo 1 se obtiene el valor más bajo de RMSEA $(0,017)$, igualmente importante para determinar la fiabilidad de un modelo. Aunque el análisis parte de modelos que muestran todos un RMSEA adecuado, inferior o igual a 0,05, el modelo 1 muestra un mejor ajuste en este sentido.

Por último, aunque no es igual de importante para determinar el mejor modelo, se puede observar cómo el valor de Chi-cuadrado es uno de los más bajos, siendo el modelo $6(64,859)$ el único que ha obtenido una puntuación inferior al modelo $1(85,546)$. Sin embargo, si nos fijamos en el P-Valor, que debe ser lo más alto posible para no resultar significativo, el modelo 1 tiene el valor más alto en P-Valor $(0,4325)$.

Cuadro 4

Resultados de los diferentes estadísticos analizados con el método ULSMV

\begin{tabular}{lcccccc}
\hline & Chi $^{2}$ & P-Value & RMSEA & Intervalo RMSA & CFI & TLI \\
\cline { 2 - 7 } Modelo 1 & 85,5 & 0,4325 & 0,017 & $0,000-0,070$ & 0,979 & 0,974 \\
Modelo 2 & 93,5 & 0,3242 & 0,031 & $0,000-0,075$ & 0,925 & 0,910 \\
Modelo 3 & 88,7 & 0,3147 & 0,032 & $0,000-0,077$ & 0,922 & 0,902 \\
Modelo 4 & 99,6 & 0,1175 & 0,053 & $0,000-0,090$ & 0,786 & 0,733 \\
Modelo 5 & 94,2 & 0,3329 & 0,030 & $0,000-0,075$ & 0,929 & 0,916 \\
Modelo 6 & 64,8 & 0,1479 & 0,055 & $0,000-0,099$ & 0,874 & 0,846 \\
\hline
\end{tabular}

Nota. Elaboración propia.

Con estos análisis podemos confirmar que nuestro instrumento se ajusta a un modelo de 2 factores, concretamente al modelo 1 donde se habían fijado todos los errores entre ítems de las mismas preguntas a 0 , pero se había dejado libre la correlación entre coche 4 y tren 2.

Por tanto, el instrumento responde o reconoce la existencia de dos factores: representación del problema y resolución del problema, considerándose dos dimensiones independientes. Respaldando asíla propuesta teórica, donde la dimensión de representación del problema (incluyendo las fases explorar y comprender; y representar y formular) implica unos procesos independientes a la dimensión de resolución del problema (incluyendo las fases planificar y ejecutar; y observar y reflexionar).

\subsection{Descripción y validación del modelo de 2 factores}

En segundo lugar, la correlación estandarizada, la cual permiten la comparación directa entre ítems, entre los dos factores definidos en el modelo elegido es de 0,969, con P-Valor significativa (0,000); lo que indica una muy buena correlación y se interpreta como algo muy positivo para la validez del instrumento. 
Además, para terminar de explicar el modelo de 2 factores, se muestran los resultados de los pesos factoriales estandarizados y sus probabilidades asociadas en el Cuadro 5.

Estos pesos factoriales son bastante adecuados, pero en el caso de Agenda 1 y Climatizador 2 las probabilidades muestran que no son significativos, en Coche 4 y Tren 2 las probabilidades son ajustadas pero se pueden considerar aceptables.

Además, en el Cuadro 5 se comprueba que existe un peso negativo en el ítem Coche 4. Según los resultados, las personas que puntúen en este ítem tendrían una menor puntuación en el factor, lo que explicaría la correlación encontrada con Tren 2 que sugiere el modelo.

Cuadro 5

Distribución de los pesos factoriales de cada item en el factor correspondiente

\begin{tabular}{lccccc}
\hline \multicolumn{1}{c}{ Factor 1 } & \multicolumn{5}{c}{ Factor 2 } \\
\hline \multicolumn{1}{c}{ Ítem } & Peso fact. & Prob. as. & Ítem & Peso fact. & Prob. as. \\
\hline Agenda 1 & 0,127 & 0,458 & Agenda 2 & 0,792 & 0,000 \\
Vídeo 1 & 0,441 & 0,004 & Vídeo 2 & 0,435 & 0,002 \\
Billetes 2 & 0,573 & 0,000 & Billetes 1 & 0,548 & 0,000 \\
Coche 1 & 0,387 & 0,005 & Billetes 3 & 0,608 & 0,000 \\
Coche 2 & 0,511 & 0,000 & Coche 3 & 0,278 & 0,031 \\
Climatizador 1 & 0,493 & 0,001 & Coche 4 & $-0,319$ & 0,136 \\
Tren 1 & 0,685 & 0,000 & Climatizador 2 & 0,085 & 0,585 \\
& & & Tren 2 & 0,322 & 0,139 \\
\hline
\end{tabular}

Nota. Elaboración propia.

Por otra parte, se expone la proporción de la varianza explicada por cada ítem a través de R-Cuadrado, cuyos porcentajes y sus probabilidades asociadas se pueden observar en el Cuadro 6, siendo agenda 1 y climatizador 2 los ítems que explican menor porcentaje de varianza, en torno a un escaso $1 \%$, y cuya probabilidad asociada es más elevada.

En este punto es importante recordar que el modelo seleccionado se comparó previamente con varios modelos de dos factores en los que se eliminaban estos ítems que menor porcentaje de la varianza explican, pero los estadísticos generales de adecuación del modelo resultaron peores que los estadísticos del modelo elegido, sin eliminar ningún ítem.

Cuadro 6

Porcentaje de varianza explicado por cada item

\begin{tabular}{lccccc}
\hline \multicolumn{1}{c}{ Factor 1 } & \multicolumn{5}{c}{ Factor 2 } \\
\hline Ítem & \% varianza & Prob. & Ítem & \% varianza & Prob. \\
\hline Agenda 1 & 1,6 & 0,711 & Agenda 2 & 62 & 0,000 \\
Vídeo 1 & 19,5 & 0,155 & Vídeo 2 & 19 & 0,126 \\
Billetes 2 & 32,8 & 0,078 & Billetes 1 & 30 & 0,071 \\
Coche 1 & 15 & 0,165 & Billetes 3 & 37 & 0,024 \\
Coche 2 & 26,1 & 0,035 & Coche 3 & 7,7 & 0,282 \\
Climatizador 1 & 24,3 & 0,092 & Coche 4 & 10,2 & 0,456 \\
Tren 1 & 46,9 & 0,013 & Climatizador 2 & 0,7 & 0,785 \\
& & & Tren 2 & 10,3 & 0,460 \\
\hline
\end{tabular}

Nota. Elaboración propia. 
Por último, en el Cuadro 7, se recoge la dificultad estimada de cada ítem según la Teoría de Respuesta al Ítem (TRI), en la que a través de una escala normalizada, donde 0 es el valor medio, un valor igual a 0 significa que para tener el 50\% de probabilidades de acertarlo debes tener una puntuación media. Esta escala pretende mostrar el nivel de constructo necesario para responder bien a cada ítem, permitiendo conocer qué ítems van a responder correctamente, aunque su competencia en RPC sea baja, y cuáles permiten distinguir a alumnos con mayor competencia.

Cuadro 7

Dificultad estimada según la TRI de cada respuesta a cada ítem

\begin{tabular}{|c|c|c|c|}
\hline \multicolumn{2}{|r|}{ Factor 1} & \multicolumn{2}{|c|}{ Factor 2} \\
\hline Ítem & Dificultad estimada & Ítem & Dificultad estimada \\
\hline Agenda 1 & R1. 0,191 / R2 1,691 & Agenda 2 & $-0,309$ \\
\hline Vídeo 1 & 0,191 & Vídeo 2 & R1. 0,038 / R2.1,335 \\
\hline Billetes 2 & R1. 0,230 / R2. 0,908 & Billetes 1 & $-0,349$ \\
\hline Coche 1 & R1. 0,431 / R2. 1,550 & Billetes 3 & $-0,114$ \\
\hline Coche 2 & R1. 0,349 / R2. 1,434 & Coche 3 & R1. -0,651 / R2. 1,876 \\
\hline Climatizador 1 & $-0,230$ & Coche 4 & 1,335 \\
\hline \multirow[t]{2}{*}{ Tren 1} & $-0,153$ & Climatizador 2 & R1. -0,230 / R2. 1,097 \\
\hline & & Tren 2 & R1. 0,967 / R2.1,691 \\
\hline
\end{tabular}

Nota. Elaboración propia.

\section{Discusión y conclusiones}

En este artículo se ha validado el constructo teórico de un instrumento en español para evaluar la aplicación de estrategias como el pensamiento computacional en la resolución de problemas (OECD, 2010), basado en el marco que propuso el programa internacional de evaluación de estudiantes PISA en 2012, y que a su vez se fundamenta en el enfoque de sistemas complejos de Greiff et al. (2012).

Hasta la fecha, la mayoría de instrumentos de evaluación del pensamiento computacional se realizan a través de programación y robótica (Tang et al., 2020). Por este motivo, el instrumento aquí presentado es una alternativa a los instrumentos hasta ahora utilizados para la evaluación del pensamiento computacional, ya que debe reclamarse una visión de este pensamiento para que realmente aporte valor más allá de la programación: en la resolución de problemas (Blackwell et al., 2008).

Este instrumento se traduce en una batería de pruebas que evalúan esta competencia, concretamente seis pruebas compuestas por 15 preguntas, válidas para muestras comprendidas entre los 14 y 16 años. Según los resultados del modelo de dos factores utilizado, la batería de pruebas creada es sólida y adecuada para evaluar el pensamiento computacional, al ser éste aplicado como una estrategia en la resolución de problemas, y que, por tanto, será un instrumento adecuado para investigar sobre estrategias empleadas en la resolución de problemas complejos en contextos educativos.

Los resultados negativos de probabilidad no significativa asociada a pesos factoriales en 3 ítems, no son concluyentes pues se ha realizado pruebas eliminando estos ítems y no mejora el modelo, de hecho el RMSEA fue superior a 0,05 , por lo que se desechó este modelo (excepto en la prueba de un factor, modelo 6). Igualmente la correlación negativa de Coche 4 , se ha ajustado estableciendo la correlación a 0 en el modelo 2 y tampoco supone una mejora de ajuste del modelo. Todos estos resultados convendrían probarlos con una muestra mayor.

Por tanto, como limitaciones del estudio, hay que destacar el bajo número de participantes de la muestra. Y como prospectiva, se continuará con la validación del instrumento con una muestra mayor para la 
misma población, y además, realizando adaptaciones de las pruebas para poder aplicarlas a diferentes edades, con el objetivo de ampliar la población de referencia.

\section{Referencias}

Blackwell, A. F., Church, L. y Green, T. R. G. (2008). The abstract is' an enemy': Alternative perspectives to computational thinking [Comunicación]. 20 Taller annual de Psychology of Programming Interest Group, Lancaster, Reino Unido.

Brennan, K. y Resnick, M. (2012). New frameworks for studying and assessing the development of computational thinking [Comunicación]. Annual meeting of the American Educational Research Association, Vancouver, Canada.

Funke, J. (2001). Dynamic systems as tools for analysing human judgement. Thinking y Reasoning, 7(1), 69-89. https://doi.org/10.1080/13546780042000046

Funke,J. (2013). Human problem solving in 2012. The Journal of Problem Solving, 6(1), 2-19. https://doi.org/10.7771/19326246.1156

Funke, J. y Frensch, P. (1995). Complex problem-solving research in North America and Europe: An integrative review. Foreign Psychology, 5, 42-47.

Gallardo-Gil, M., Fernández-Navas, M., Sepúlveda-Ruiz, M. P., Serván, M. J., Yus, R. y Barquín, J. (2010). PISA y la competencia científica: Un análisis de las pruebas de PISA en el Área de Ciencias. RELIEVE, 16(2), 1-17. https://doi.org/10.7203/relieve.16.2.4138

González-Mayorga, H., Vidal, J. y Vieira, M. J. (2017). El impacto del Informe PISA en la sociedad española: El caso de la prensa escrita. Revista Electrónica de Investigación y Evaluación Educativa, 23(1), 1-17. https://doi.org/10.7203/relieve.23.1.9015

Greiff, S., Holt, D. V. y Funke, J. (2013). Perspectives on problem solving in educational assessment: Analytical, interactive, and collaborative problem solving. Journal of Problem Solving, 5(2), 71-91.

Greiff, S., Wüstenberg, S. y Funke, J. (2012). Dynamic problem solving: A new assessment perspective. Applied Psychological Measurement, 36(3), 189-213. https://doi.org/10.1177/0146621612439620

Greiff, S., Wüstenberg, S., Molnár, G., Fischer, A., Funke, J. y Csapó, B. (2013). Complex problem solving in educational contexts. Something beyond g: Concept, assessment, measurement invariance, and construct validity. Journal of Educational Psychology, 105(2), 364-379. https://doi.org/10.1037/a0031856

Heintz, F., Mannila, L. y Färnqvist, T. (2016). A review of models for introducing computational thinking, computer science and computing in K-12 education [Comunicación]. Frontiers in Education Conference (FIE), Erie, Estados Unidos de América.

Herde, C. N., Wüstenberg, S. y Greiff, S. (2016). Assessment of complex problem solving: What we know and what we don't know. Applied Measurement in Education, 29(4), 265-277.

https://doi.org/10.1080/08957347.2016.1209208

Hsu, T. C., Chang, S. C. y Hung, Y. T. (2018). How to learn and how to teach computational thinking: Suggestions based on a review of the literature. Computers \& Education, 126, 296-310.

Huggins-Manley, A. C. y Algina, J. (2015). The partial credit model and generalized partial credit model as constrained nominal Rresponse models, with applications in Mplus. Structural Equation Modeling: A Multidisciplinary Journal, 22(2), 308-318.

https://doi.org/10.1080/10705511.2014.937374

Koh, K. H., Nickerson, H., Basawapatna, A. y Repenning, A. (2014). Early validation of computational thinking pattern analysis [Comunicación]. 2014 Conference on Innovation and Technology in Computer Science Education, Colorado, Estados Unidos de América.

Kwisthout, J. (2012). Relevancy in problem solving: A computational framework. The Journal of Problem Solving, 5(1), 18-33. 
Li, C. H. (2014). The performance of MLR, USLMV, and WLSMV estimation in structural regression models with ordinal variables. Michigan State University.

Lye, S. Y. y Koh, J. H. L. (2014). Review on teaching and learning of computational thinking through programming: What is next for K-12?. Computers in Human Behavior, 41, 51-61.

OECD (2010). PISA 2012 field trial problem solving framework: Draft subject to possible revision after the field trial. OECD. Recuperado de http://www.oecd.org/pisa/pisaproducts/46962005.pdf.

Olea, J. y Ponsoda, V. (1998). Tests informatizados y adaptativos informatizados: investigación en España. Revista Electrónica de Investigación y Evaluación Educativa, 4(2), art1.

Ortega-Ruipérez, B. (2018). Pensamiento computacional y resolución de problemas (Tesis doctoral). Universidad Autónoma de Madrid. UAM

Ortega-Ruipérez, B. y Asensio, M. (2018). Robótica DIY: Pensamiento computacional para mejorar la resolución de problemas. RELATEC: Revista Latinoamericana de Tecnología Educativa, 17(2), 129-143.

Román, M. y Murillo, F. J. (2009). La evaluación de los aprendizajes escolares: Un recurso estratégico para mejorar la calidad educativa, Revista Iberoamericana de Evaluación Educativa, 2(1), 4-9.

Román-González, M., Pérez-González, J. C., Moreno-León. y J., Robles, G., (2018). Can computational talent be detected? Predictive validity of the computational thinking test. International Journal of Child-Computer Interaction, 18, 47-58.

Schleicher, A. (2006). Fundamentos y cuestiones políticas subyacentes al desarrollo de PISA. Revista de Educación, 26, 21-43.

Schoppek, W. (2002). Examples, rules, and strategies in the control of dynamic systems. Cognitive Science Quarterly, 2(1), 63-92.

Tang, X., Yin, Y., Lin, Q., Hadad, R. y Zhai, X. (2020). Assessing computational thinking: A systematic review of empirical studies. Computers \& Education, 148, 103798.

https://doi.org/10.1016/j.compedu.2019.103798

Wing, J. (2006). Computational thinking. Communications of the ACM, 49, 33-35.

Wing, J. (2014). Computational thinking benefits society. Academic Press.

Zapata-Ros, M. (2015). Pensamiento computacional: Una nueva alfabetización digital. RED Revista de Educación a Distancia, 46(4). 


\section{Anexo I}

\section{Inicio}

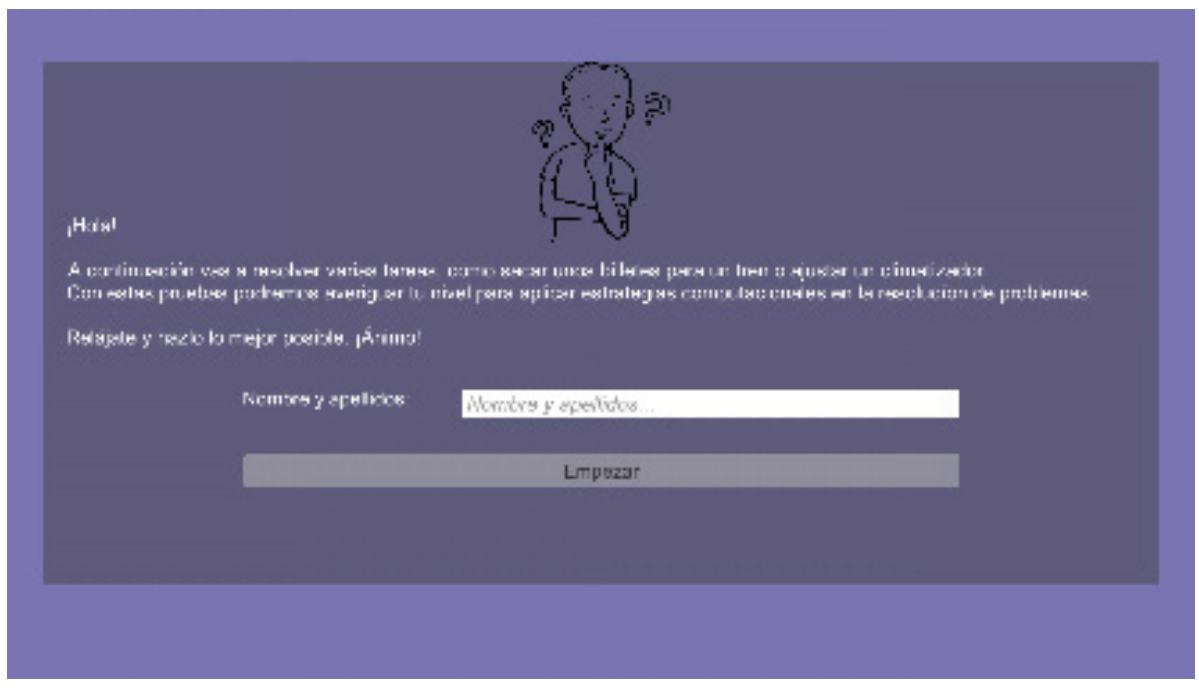

\section{Prueba Agenda}
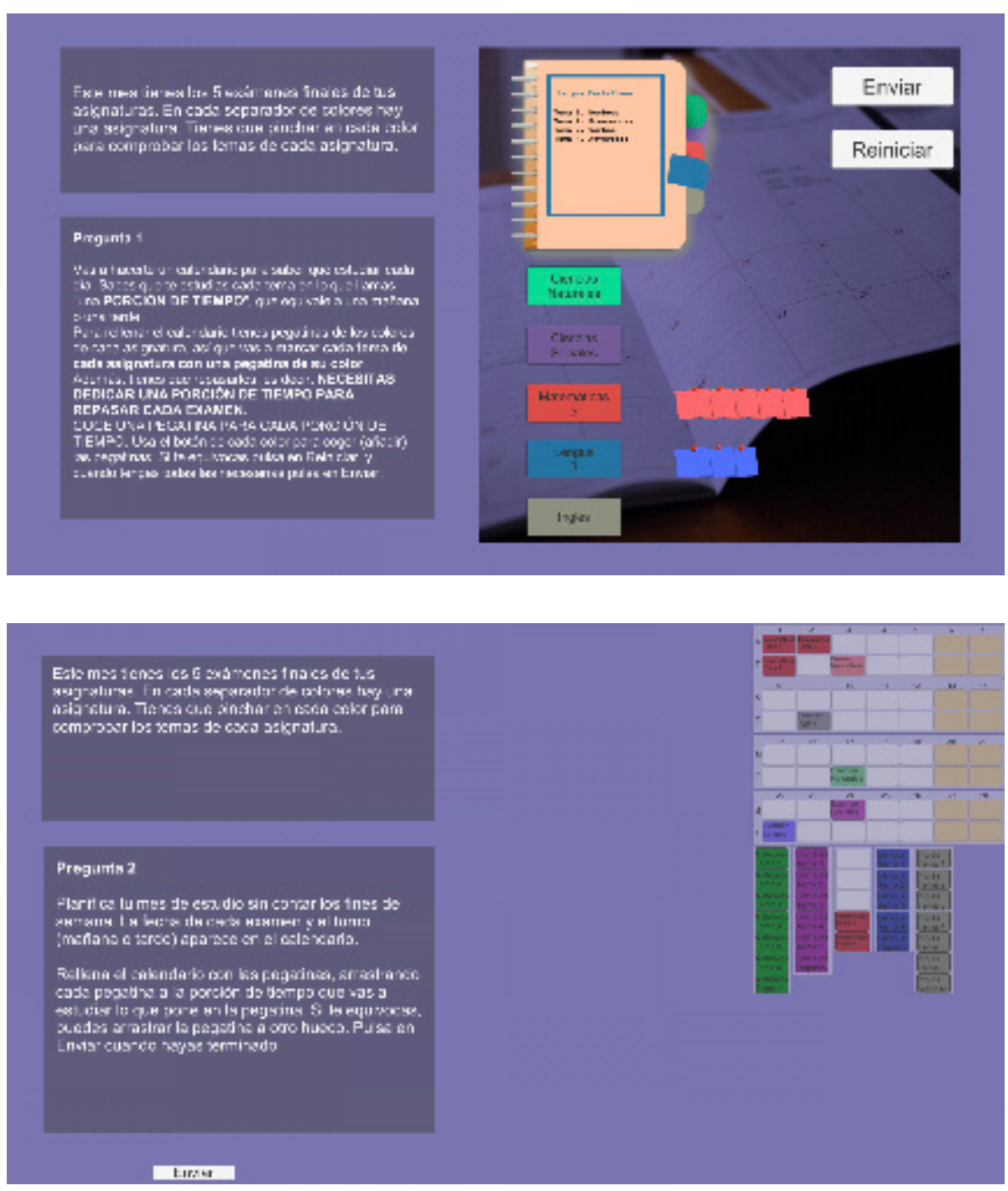


\section{Prueba Vídeo}

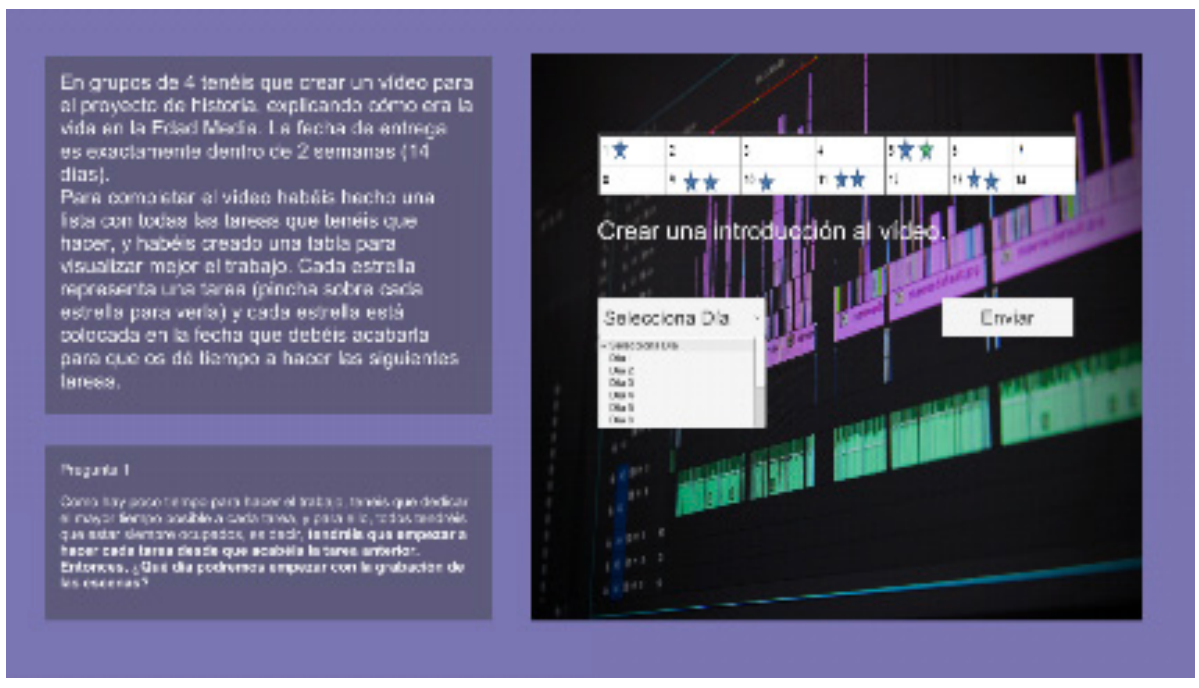

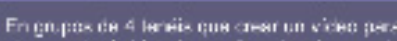

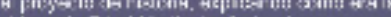

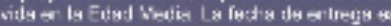

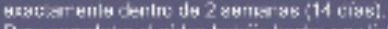

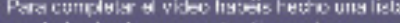

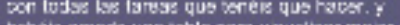

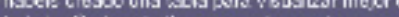

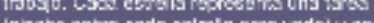
iprens edoxe cads esirelo pas benal ; case saticie cra co cesca en a fests que cebsi

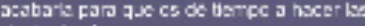
equemas tercas.

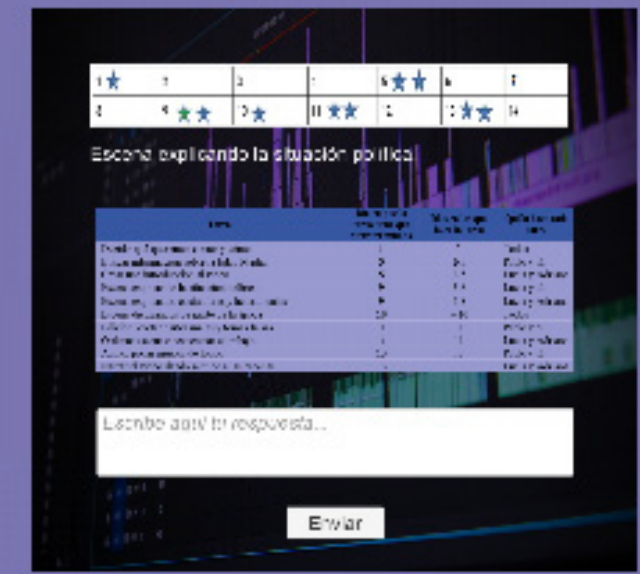

$\rightarrow \infty-\infty$

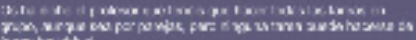

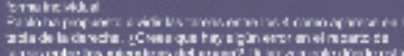
in

\section{Prueba Billetes (Pruebas PISA)}

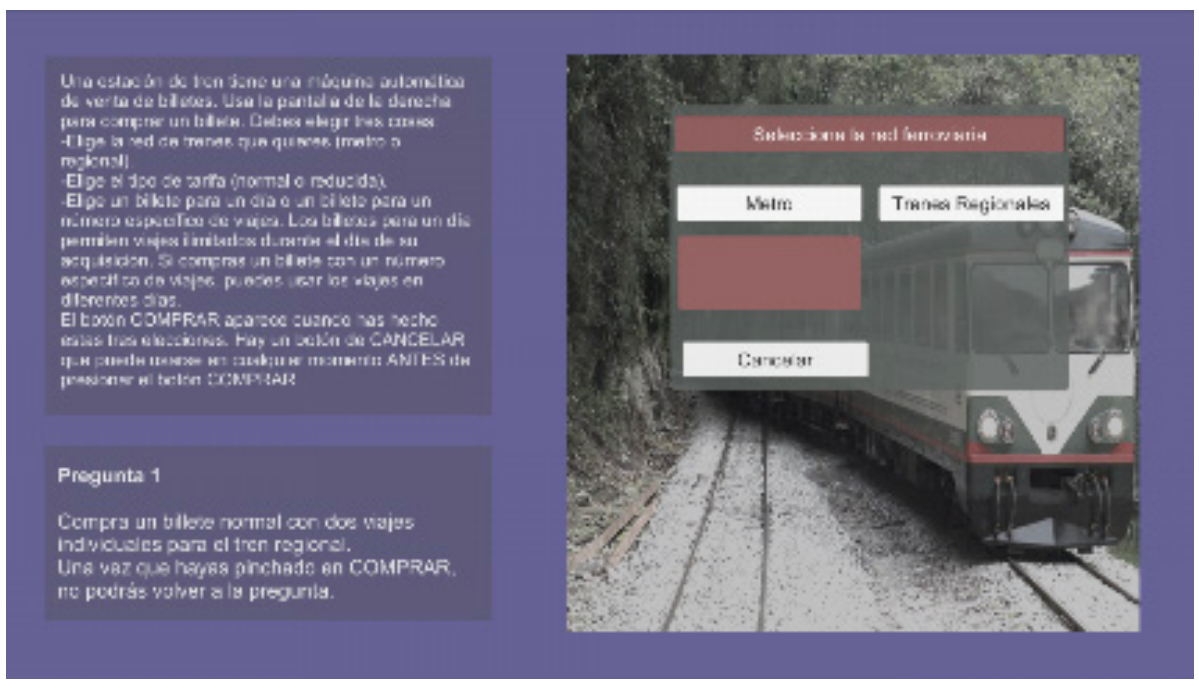




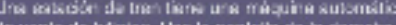

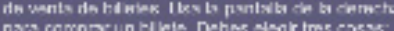
pas comprorun El leit. Dabes slagr tres cosas: magres:

(1)

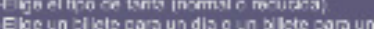

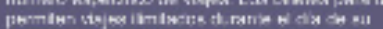

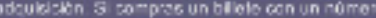
espseriso de va a ea, pusdez asr ke vízses en (1)

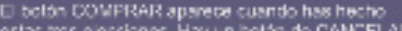

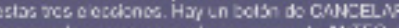

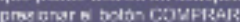

Progunts 2

llay stsness haser custo wisise por la chusas en

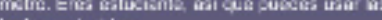

Ir tas wau wave

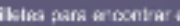
.

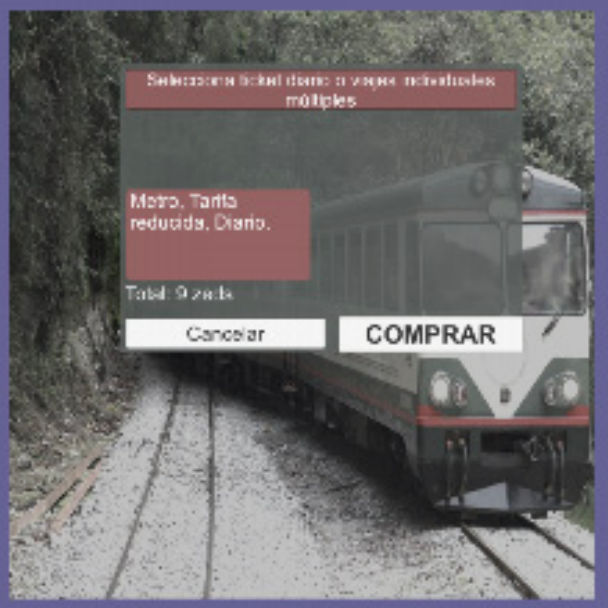

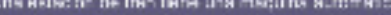

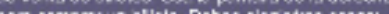

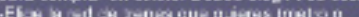

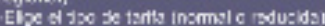

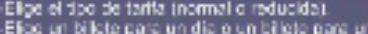

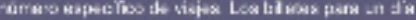

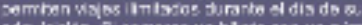

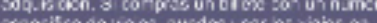

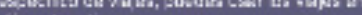

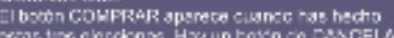

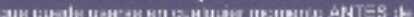
smekener $w$ bstho Cotarials

\section{Progunta 3}

Quers osmera un tilets de matro urbans cen cos

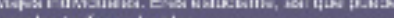

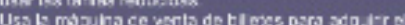
Uss is mapunace verts

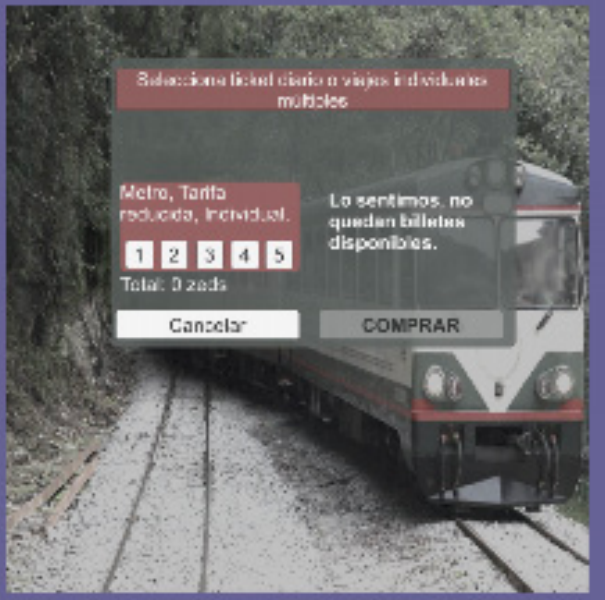

\section{Prueba Tren}

Nos vamos de viaje! Este verano vamos a hacer un interrail por

Europa, viajando en tren por un

montón de paises.

El mapa de la derecha muestra las horas que se lardan viajando enlru

los destinos señalados.
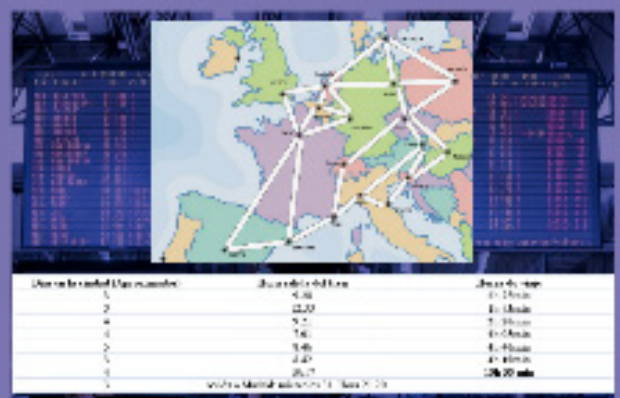

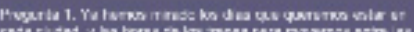

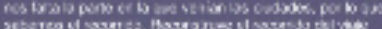

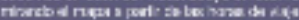

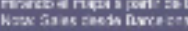

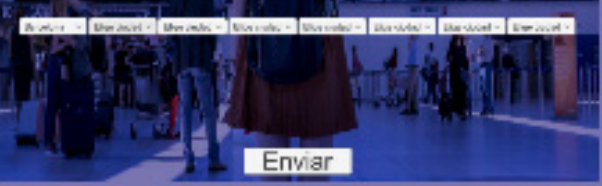




\section{Prueba Climatizador (Pruebas PISA)}
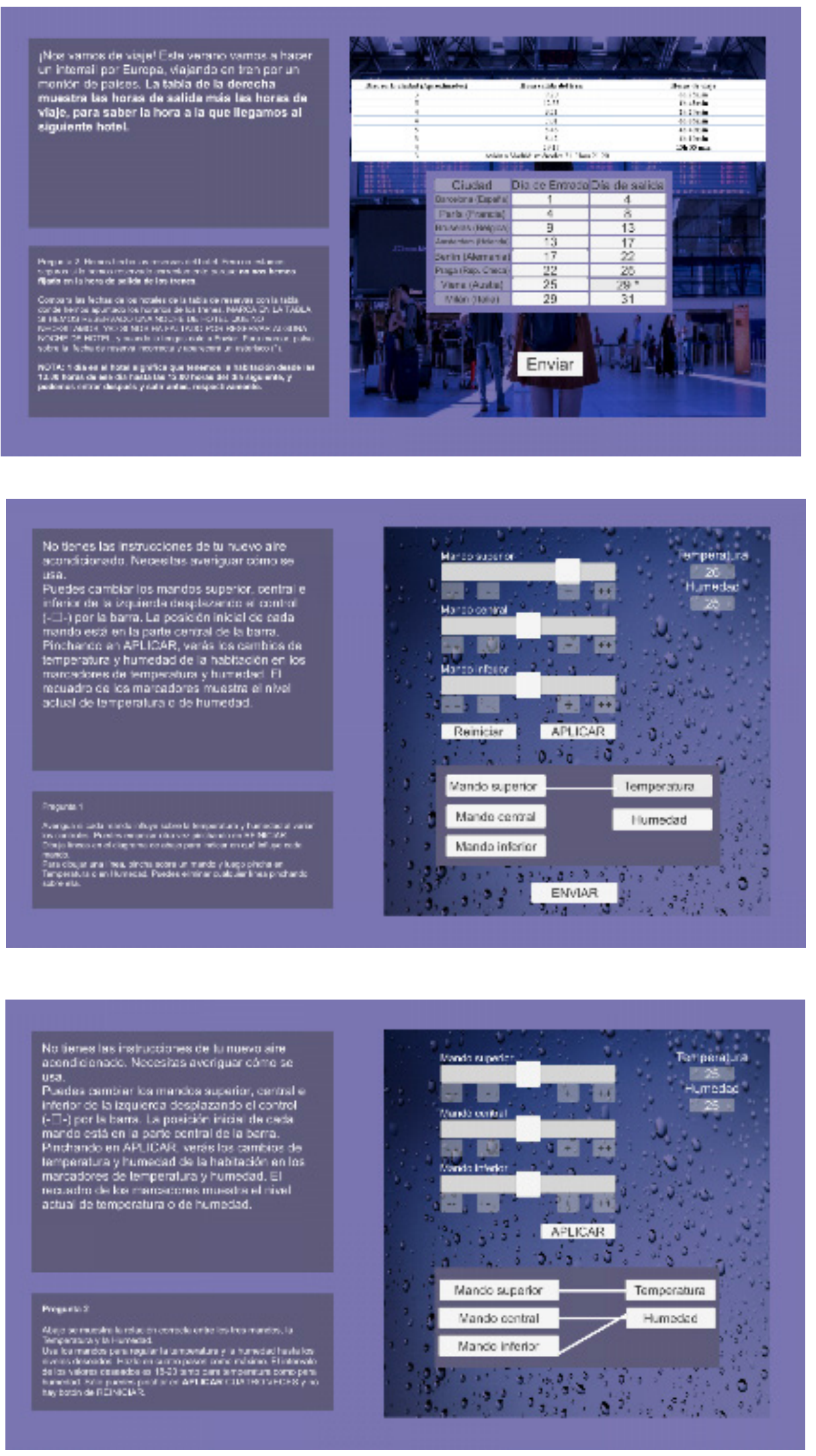


\section{Prueba Coche}

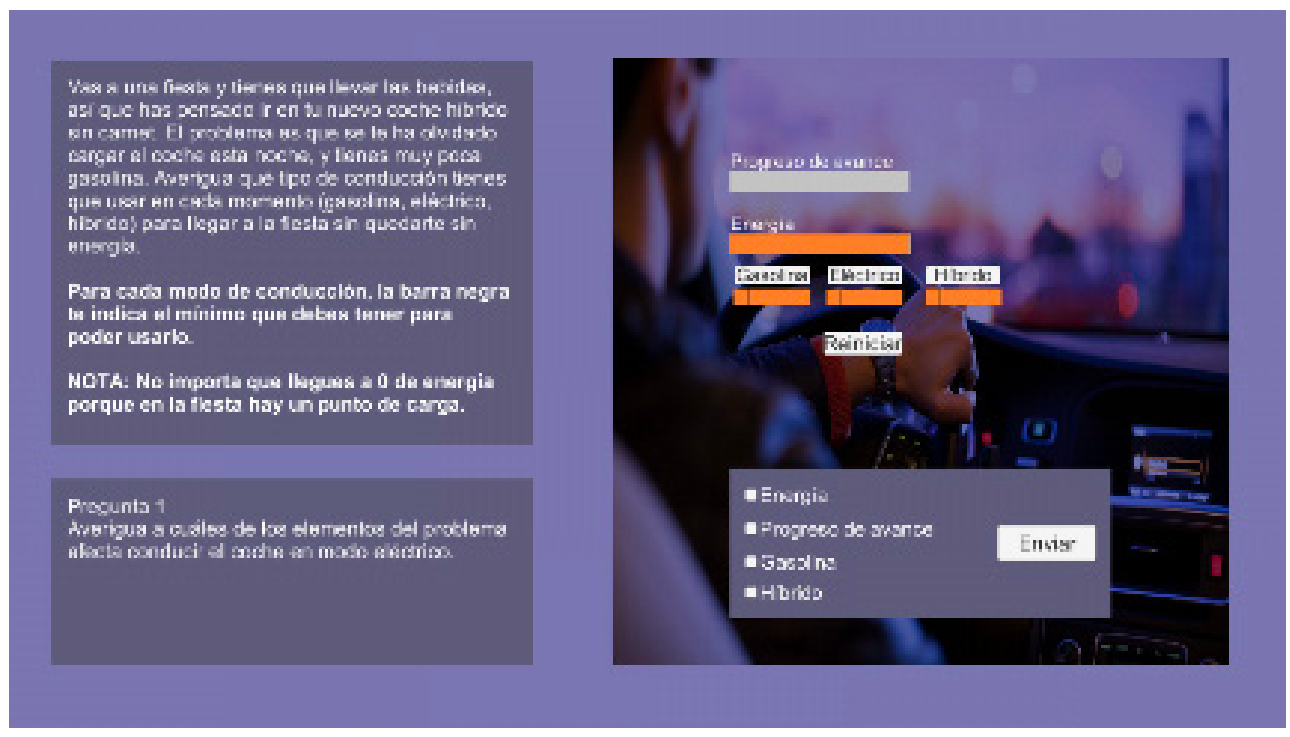

Vas a una festa y jerca cue llera as bebidas. sel qua has psosesce ren ta ruseo cosho ribnse

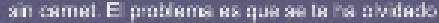
esras cl coshe cesd nochs, y tisnes m.sy sces

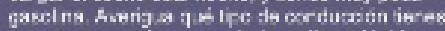
que Laar en cada mamenis igasstra, sléctriza, hib kso) par ilszar a a testa sin quadure sin annirgis.

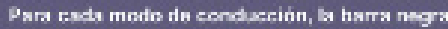
we indica el minime que delesa tener para poder usarlo.

NOTA: No importa que lkguss a o de smsrgia porque en la tiesta hay un perata ch cargs.

Preques

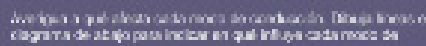

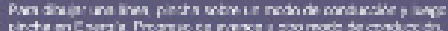

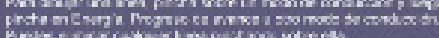

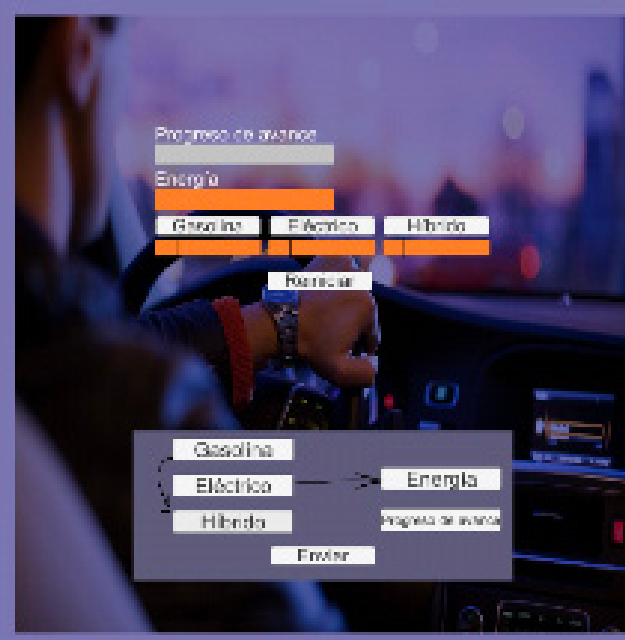

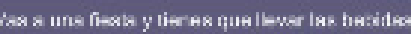
aer que has perresed r cn tu nuswa coshe hibtice

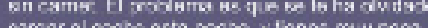

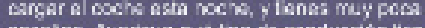

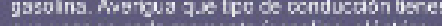

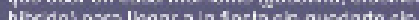
hiar cal paro isgai a
nnergla. nororg:

The marase que lapuea a C da enargis porque en la iesta nay un punto ce cams.

Prosunta 2

Imanta lkgar a ia festa. Fa:a el b, landras qua ocmpeta la surra ce progress ce soance sr yuestrit in wnergls

Si no has podido terminar, puta enviar.

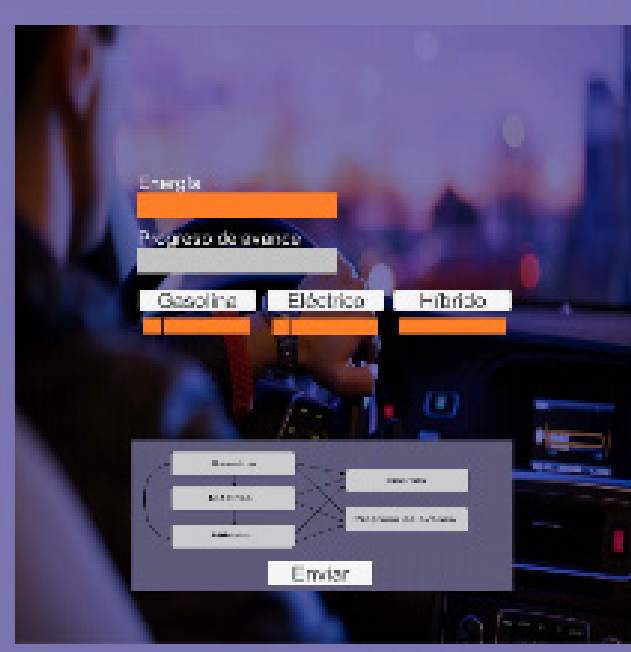




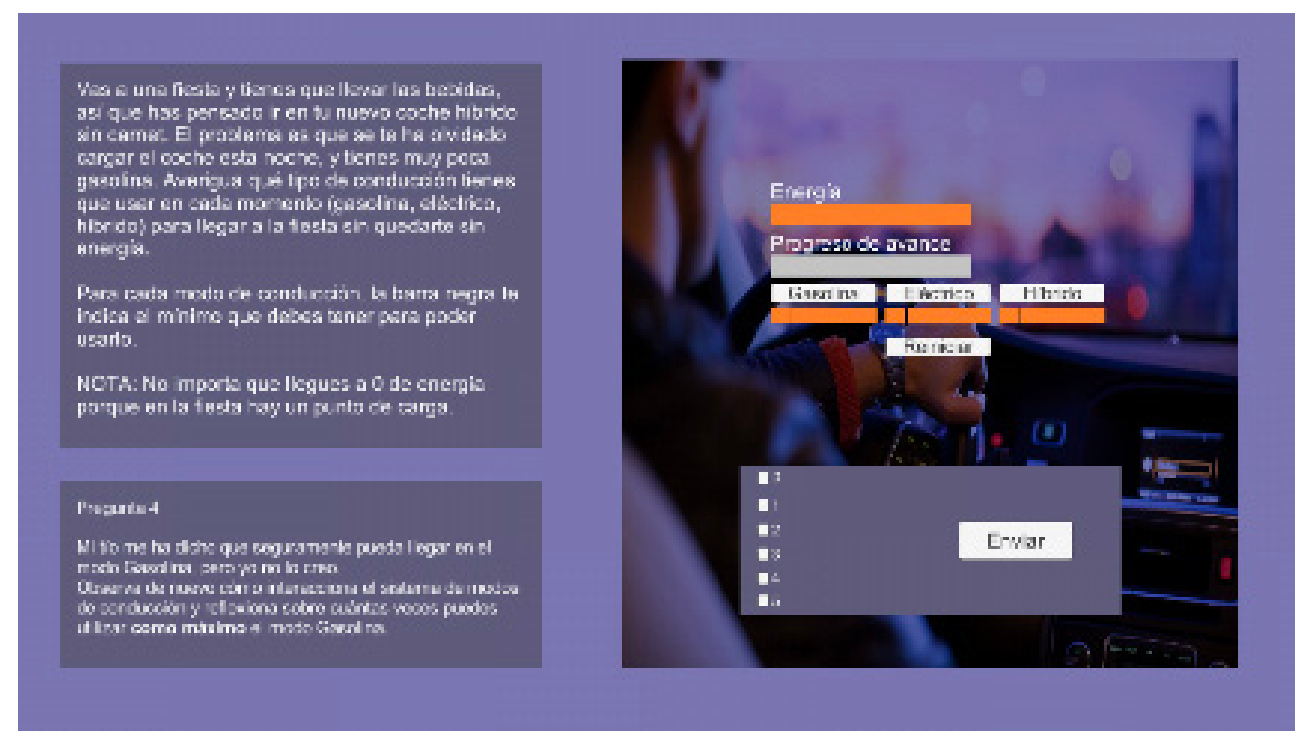

\section{Breve CVs de los autores}

\section{Beatriz Ortega Ruipérez}

Doctora en Psicología Básica (Universidad Autónoma Madrid), aplicada a la educación y en concreto a la tecnología educativa. Docente en el Máster de Tecnología Educativa de UNIR y en el Máster de Profesorado de URJC. Entre las principales líneas de investigación destacan: la estructura y evaluación del pensamiento computacional, la robótica y la programación para desarrollar este pensamiento, el diseño pedagógico de videojuegos, la inclusión de técnicas de impacto social como Aprendizaje-Servicio, y la autorregulación del aprendizaje. Email: beatriz.ortega.ruiperez@unir.net

ORCID ID: https://orcid.org/0000-0002-3822-5745

\section{Mikel Asensio Brouard}

Profesor titular e investigador en la Universidad Autónoma de Madrid desde el año 1985. Premio Nacional de Investigación. Ha sido Vicedecano, Delegado del Rector, y durante más de 5 años Director del Museo de Artes y Tradiciones Populares de la UAM. Visitor Research y profesor invitado en varias universidades. Ha impartido numerosas conferencias en Estados Unidos, China, Europa e Iberoamérica. En Europa, ha participado en cinco proyectos europeos del quinto, sexto y séptimo programa marco. Ha sido director de investigaciones competitivas nacionales y regionales. $Y$ un centenar de proyectos en el ámbito profesional del patrimonio. Ha dirigido 11 tesis doctorales. Es autor de 261 publicaciones en temas de Museología y Patrimonio, Educación y Psicología. Email: mikel.asensio@uam.es.

ORCID ID: https://orcid.org/0000-0002-1020-5486 